\title{
ON THE MASS-TO-LIGHT RATIOS FOR DOUBLE GALAXIES
}

\author{
R. J. DICKENS \\ Royal Greenwich Observatory, Herstmonceux, Great Britain \\ and \\ J. V. PEACH \\ Dept. of Astrophysics, University of Oxford, Great Britain
}

\begin{abstract}
Statistical mass-to-light ratios are found for spiral and irregular galaxies, and $E$ and S0 galaxies, using 57 multiple systems including 43 pairs. The results are consistent with those of the earlier study by Page. Preliminary conclusions point out the great uncertainties in such mass-to-light ratios due to inaccurate magnitudes and relative radial velocities. The derived masses are relatively insensitive to the choice of the distribution function of projected separations.
\end{abstract}

The critical importance of galaxy masses and mass-to-light ratios for studies of their stellar content and for the resolution of such problems as the stability of groups and clusters of galaxies makes any observational evidence bearing on the masses of galaxies, and especially E and S0 galaxies particularly valuable. There is little information available on the masses of ellipticals from rotation curves, and only slightly more from the observationally difficult measurements of internal velocity dispersion. The use of the double galaxy method appears competitive with these, and we have reinvestigated the method in the light of two advances which suggest that such a reinvestigation is now opportune. These are, firstly, that since the work of Page (1952, 1966) and Holmberg (1954) there are now more velocities available for double and multiple systems, and secondly, that since the publication of Zwicky's Catalogue of Galaxies and Clusters of Galaxies it is possible to redetermine the frequency function of space separations of physical pairs, which is needed in the reduction of the radial velocity differences and angular separations to give masses.

\section{Outline of the Method}

Assuming circular orbits and point masses we can write an average over all pairs

$$
\overline{r v^{2}}=8 \pi^{2} \bar{M} \overline{\cos ^{3} x} \overline{\cos ^{2} y}
$$

where $r$ is the projected linear separation, $v$ the differential radial velocity, $2 M$ the mass of the pair, and $x$ and $y$ are the angles between the radius vector and the celestial sphere and between the direction of orbital motion and a plane containing the radius vector and the Sun respectively. For a random distribution of $x$ and $y, \overline{\cos ^{3} x}=3 \pi / 16$ and $\overline{\cos ^{2} y}=\frac{1}{2}$. While the distribution of $y$ is certainly random, that of $x$ depends on the actual distribution of true space separations between physical pairs $F(R)$. Now it 
can be shown that

$$
f(r)=\int_{r}^{R_{m}} \frac{r F(R) \mathrm{d} R}{R \sqrt{R^{2}-r^{2}}},
$$

where $f(r)$ is the number distribution of physical pairs as a function of projected separation and $R_{m}$ is the maximum separation of physical pairs. $f(r)$ can be determined from counts of galaxies around selected central galaxies chosen at random with an appropriate correction for optical companions. Then we can write

$$
\overline{v^{2}}=4 \pi^{2} \bar{M} g\left(r, R_{m}\right)
$$

where $g\left(r, R_{m}\right)$ contains integrals involving $F(R)$. A regression of $v^{2}$ on $g\left(r, R_{m}\right)$ leads to $\bar{M}$.

\section{Determination of $F(R)$}

The distribution of physical pairs around galaxies with known velocities and with $m_{p g} \leqslant 13.0$ was determined as follows. The positions of all galaxies with $m_{p g} \leqslant 15.5$ in circles of $2^{\circ}$ radius centred on these galaxies were taken from Zwicky's Catalogue and their distances from the central galaxies calculated assuming that they had the same modulus, the modulus being computed from the velocity with $H_{0}=$ $100 \mathrm{~km} \mathrm{~s}^{-1} \mathrm{Mpc}^{-1}$. The number of galaxies as a function of true separation is determined by combining data from all $(\sim 300)$ fields with appropriate correction for optical companions, assuming the distribution of these on the celestial sphere is random. The excess over a random distribution gives the distribution of projected separations of physical pairs, $f(r)$ from which $F(R)$ is obtained by deprojection. Hence $g\left(r, R_{m}\right)$ can be calculated for application to the pair velocity data.

\section{Radial Velocities and Angular Separations}

All galaxies with published radial velocities were investigated for possible physical companions; both pure pairs and multiple systems were isolated, the multiple systems consisting of more than two galaxies which could be treated as dynamical pairs. It proved possible to assemble reliable velocities for about $60 \%$ more systems than were considered in the treatment of double galaxy masses by Page (1966). Weights were assigned to the velocity differences on a uniform system. Angular separations were measured from the Sky Survey prints. The photographic magnitudes were taken from the Reference Catalogue of Bright Galaxies (de Vaucouleurs and de Vaucouleurs, 1964) and corrected for a galactic absorption of 0.24 mag at the poles.

\section{Results and Discussion}

Preliminary results of least squares solutions for various groups of the data are given in Table I where they are compared with the results of Page (1966). Masses and $M / L$ 
TABLE I

Masses and mass-to-light ratios for multiple systems $\left(H_{0}=100 \mathrm{~km} \mathrm{~s}^{-1} \mathrm{Mpc}^{-1}\right)$

\begin{tabular}{|c|c|c|c|c|c|}
\hline & & \multirow{2}{*}{$\begin{array}{l}\text { Pure pairs only } \\
\text { Page (1966) }\end{array}$} & \multirow[b]{2}{*}{$\begin{array}{l}\text { This } \\
\text { investigation }\end{array}$} & \multicolumn{2}{|c|}{ Pure + multiple systems } \\
\hline & & & & Page (1966) & $\begin{array}{l}\text { This } \\
\text { investigation }\end{array}$ \\
\hline SP, IRR & $\left\{\begin{array}{l}10^{-10} M / M_{\odot} \\
M / L \text { (solar units) } \\
\text { number of systems }\end{array}\right.$ & $\begin{array}{l}1.6 \pm 2.3 \\
1.4 \pm 1.8 \\
10\end{array}$ & $\begin{array}{l}2.3 \pm 4.1 \\
3.0 \pm 5.4 \\
28\end{array}$ & $\begin{array}{l}4.0 \pm 4.2 \\
3.2 \pm 4.2 \\
16\end{array}$ & $\begin{array}{l}5.9 \pm 7.6 \\
7.0 \pm 9.0 \\
36\end{array}$ \\
\hline E, S0 & $\left\{\begin{array}{l}10^{-10} M / M_{\odot} \\
M / L \text { (solar units) } \\
\text { number of systems }\end{array}\right.$ & $\begin{array}{l}64 \pm 38 \\
92 \pm 92 \\
13\end{array}$ & $\begin{array}{l}44 \pm 20 \\
60 \pm 27 \\
15\end{array}$ & $\begin{array}{l}66 \pm 29 \\
98 \pm 68 \\
18\end{array}$ & $\begin{array}{l}45 \pm 14 \\
42 \pm 13 \\
21\end{array}$ \\
\hline
\end{tabular}

ratios for $\mathrm{S}$ and Irr systems are essentially unchanged. The large $M / L$ ratio for $\mathrm{E}$ galaxies in pairs should be noted as it seems difficult to reconcile with the smaller $(\sim 50)$ values obtained from other dynamical methods and with the larger $(\sim 800)$ values obtained from the application of the virial theorem to clusters of galaxies. The discrepancies in the $M / L$ ratios can partly be ascribed to a systematic difference of about 0.2 mag between the magnitudes of the Reference Catalogue and those used by Page.

A detailed account of this investigation will be published elsewhere. We should like to point out the following preliminary conclusions. Firstly it is not generally realised how great are the uncertainties in $M / L$ ratios determined by this type of statistical analysis due to the generally inaccurate magnitudes available. These are such as to render the total luminosities and hence $M / L$ ratios uncertain by about a factor two and $M / L$ is probably systematically too large. Secondly, velocity differences based on individual velocity determinations are of such low weight as compared with those based on simultaneous observation of both objects that their influence on the solutions is small and leads to larger errors. Thirdly, the derived masses are relatively insensitive to the choice of $F(R)$.

\section{References}

Holmberg, E.: 1954, Medd. Lunds Astron. Obs. Ser. I, 186, 1.

Page, T. L.: 1952, Astrophys. J. 116, 63.

Page, T. L.: 1966, in Proc. Fifth Berkeley Symposium on Mathematical Statistics and Probability, Univ. of California Press, Berkeley, 3, 31.

Vaucouleurs, G. de and Vaucouleurs, A. de: 1964, in Reference Catalogue of Bright Galaxies, Univ. of Texas Press, Austin.

\section{Discussion}

Lewis: In NGC 253-7 (my observations) and 3226-7 (Rubin and Ford) it is seen that the position angle of the major axis of the less massive galaxy is very near to the position vector joining the two galaxies. This seems to suggest that the less massive galaxy is approximately in its own plane of symmetry. If this were generally true, it suggests that the distribution of position vectors between the two galaxies and the orbits on the plane of the sky are related. This may mean that some of the quantities you assume to be independent and randomly distributed are not in fact so. 
Dickens: While this effect may be generally present, one cannot base an analysis on two cases only. If these distributions prove likely to be related by further observations, such correlations could be incorporated into the theory. However, the large errors already inherent in the method would not appear to justify consideration of such effects, at least for the present.

King: (1) Does your method involve the assumption that the orbits are circular? (2) Did you derive a mean $M / L$ directly, or did you divide a mean $M$ by a mean $L$ ?

Dickens: (1) Yes. However, Page has shown that even the assumption of purely radial orbits should not change the mean masses by more than a factor of two.

(2) The results shown used a mean $L$ for each group to obtain a mean $M / L$. However, we intend finally to solve separately the regression curve for $M / L$. 